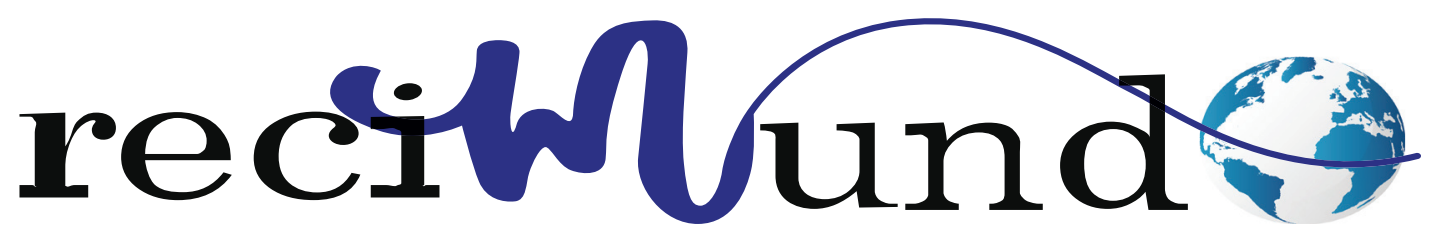

Revista Científica Mundo de la Investigación y el Conocimiento

DOI: $10.26820 /$ recimundo/5.(4).oct.2021.284-294

URL: https://recimundo.com/index.php/es/article/view/986

EDITORIAL: Saberes del Conocimiento

REVISTA: RECIMUNDO

ISSN: 2588-073X

TIPO DE INVESTIGACIÓN: Artículo de investigación

CóDIGO UNESCO: 32 Ciencias Médicas

PAGINAS: 284-294

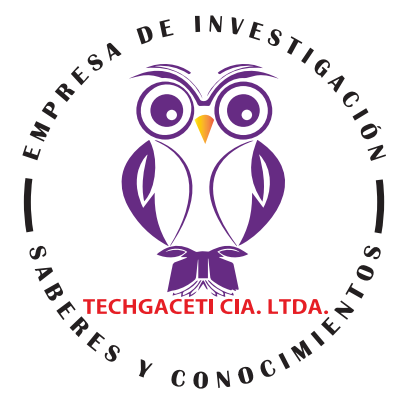

\title{
Impulsividad, monitoreo y relación parental entre adolescentes estudiantes y adolescentes usuarios de drogas
}

Impulsiveness, monitoring, and parental relationship between adolescent students and adolescent drug users

Impulsividade, monitoramento e relacionamento parental entre adolescentes estudantes e adolescentes usuários de drogas

Cecilia Colunga-Rodríguez'; Jorge Alberto Valadez-García2; Roberto Oropeza-Tena3; Mario Ángel-González;; Julio César Vázquez-Colunga5; Claudia Liliana Vázquez-Juárez ${ }^{6}$; Bertha Alicia Colunga-Rodríguez?

\section{RECIBIDO: 09/11/2021 ACEPTADO: 19/11/2021 PUBLICADO: 26/11/2021}

1. Instituto Mexicano del Seguro Social; Centro Universitario de Ciencias de la Salud, Universidad de Guadalajara. Jalisco, México. cecilia.colunga@imss.gob.mx; (iD https://orcid.org/0000-0003-0328-788X

2. Centro Universitario de Ciencias de la Salud, Universidad de Guadalajara. Sierra Nevada 950, Col. Independencia, C.P. 44340, Guadalajara Jalisco, México. jalvaladez1@gmail.com; (D) https://orcid.org/0000-0002-0503-0060

3. Universidad Michoacana de San Nicolás de Hidalgo. Michoacán, México. roberto.oropeza@umich.mx; https://orcid. org/0000-0002-2561-2164

4. Centro Universitario de Tonalá, Universidad de Guadalajara. Jalisco, México. mario.angel@academicos.udg.mx; (DD https:// orcid.org/0000-0002-5762-5170

5. Centro Universitario de Tonalá, Universidad de Guadalajara. julio.vazquez@academico.udg.mx; (iD) https://orcid.org/00000001-8393-2505

6. Centro Universitario de Tonalá, Universidad de Guadalajara. Jalisco, México. claudia.vazquezjuarez@ensj.edu.mx; (iD https://orcid.org/0000-0002-0932-0110

7. Escuela Normal Superior de Jalisco. Jalisco, México. bertha.colunga@ensj.edu.mx; iD https://orcid.org/0000-0002-25769225

\section{CORRESPONDENCIA}

Cecilia Colunga-Rodríguez

cecilia.colunga@imss.gob.mx

Jalisco, México 


\section{RESUMEN}

El objetivo de la presente investigación fue comparar los niveles de la relación padres-hijos, monitoreo parental e impulsividad entre adolescentes estudiantes sin consumo de drogas y adolescentes usuarios de drogas en tratamiento. El estudio tuvo un diseño comparativo y de corte transversal y participaron 90 adolescentes, 45 usuarios de drogas con edad promedio de 15.40 años ( $D E=.539)$ y 45 estudiantes con edad promedio de 15.26 años $(\mathrm{DE}=1.07)$. Se aplicaron tres escalas estandarizadas en adolescentes mexicanos: relación con padre y madre, monitoreo paterno e impulsividad. Para el análisis de datos se utilizó la t de Student para muestras independientes. Se encontraron diferencias estadísticamente significativas en las dimensiones comunicación paterna, comunicación materna, control paterno, control materno, monitoreo paterno, monitoreo materno e impulsividad. En los jóvenes que consumen drogas se encontró mayor impulsividad, bajo nivel de monitoreo paterno y menores puntajes en comunicación y control del padre y la madre. A diferencia de otros estudios en esta investigación no se encontró una diferencia en la dimensión de afecto tanto paterno como materno.

Palabras clave: Adolescencia, drogas, impulsividad, monitoreo paterno, relaciones padres-hijos.

\section{ABSTRACT}

The present investigation sought to compare the levels of the parent-child relationship, parental monitoring and impulsivity amongst student teenagers without drug using and drug using teenagers in treatment. The study had a comparative and cross-sectional design and 90 teenagers participated, 45 drug users with an average age of 15.40 years $(S D=.539)$ and 45 students with an average age of 15.26 years $(S D=1.07)$. Three standardized scales in Mexican teenagers were applied: relationship with father and mother, parental monitoring and impulsivity. Student's t test for independent samples was used to the data analysis. Statistically significant differences were found in the dimensions of parental communication, maternal communication, parental control, maternal control, paternal monitoring, maternal monitoring and impulsivity. In young people who use drugs, higher impulsivity, low level of parental monitoring and low communication and control scores of the father and other were found. Unlike other studies in this research, no difference was found in the dimension of both paternal and maternal affection.

Keywords: Drugs, impulsivity, parental monitoring, parent-child relationship, teenagers.

\section{RESUMO}

A presente investigação buscou comparar os níveis de relação pais-filhos, monitoramento parental e impulsividade entre adolescentes estudantes sem uso de drogas e adolescentes usuários de drogas em tratamento. O estudo teve um desenho comparativo e transversal e contou com a participação de 90 adolescentes, 45 usuários de drogas com idade média de 15,40 anos ( $d p=0,539)$ e 45 estudantes com idade média de 15,26 anos ( $d p=1,07)$. Três escalas padronizadas em adolescentes mexicanos foram aplicadas: relacionamento com pai e mãe, monitoramento parental e impulsividade. 0 teste t de Student para amostras independentes foi usado para a análise dos dados. Diferenças estatisticamente significativas foram encontradas nas dimensões de comunicação parental, comunicação materna, controle parental, controle materno, monitoramento paterno, monitoramento materno e impulsividade. Em jovens usuários de drogas, constatou-se maior impulsividade, baixo nível de monitoramento dos pais e baixos escores de comunicação e controle do pai e de outras pessoas. Ao contrário de outros estudos desta pesquisa, não foi encontrada diferença na dimensão do afeto paterno e materno.

Palavras-chave: Drogas, impulsividade, monitoramento parental, relação pai-filho, adolescentes. 
COLUNGA-RODRÍGUEZ, C., VALADEZ-GARCÍA, J., OROPEZA-TENA, R., ÁNGEL-GONZÁLEZ, M., VÁZQUEZ-COLUNGA, J., VÁZQUEZ-JUÁREZ, C., \& COLUNGA-RODRÍGUEZ, B.

\section{Introducción}

De acuerdo a la Encuesta Nacional de Consumo de Drogas en Estudiantes (ENCODE) realizada en el 2014, se reportó que a nivel nacional el $18.8 \%$ de los estudiantes de preparatoria eran fumadores actuales (Villatoro-Velázquez et al., 2015a), por su lado, la prevalencia del consumo de alcohol en estudiantes de secundaria y bachillerato fue de $53.2 \%$ (Villatoro-Velázquez et al., 2015b), mientras que el porcentaje de estudiantes de secundaria y bachillerato que alguna vez consumieron cualquier droga ilegal fue de $17.2 \%$ en total (Villatoro-Velázquez et al., 2015c).

Los datos epidemiológicos nos indican la importancia de ampliar el conocimiento sobre los factores que influyen para que un adolescente inicie el consumo de sustancias psicoactivas (SPA), en ese sentido se ha encontrado que las relaciones sociales con otros jóvenes que consumen drogas y las relaciones familiares disfuncionales son los factores de riesgo de mayor impacto para iniciar el consumo de SPA (Figueroa-Varela et al., 2019). En una investigación donde se entrevistó a expertos en prevención de las adicciones refirieron que en el ámbito de lo familiar los factores de riesgo más significativos son la insuficiente supervisión familiar y la deficiente disciplina familiar (Valadez et al., 2018).

En términos de factores protectores, la familia es uno de los principales contextos en donde los adolescentes adquieren recursos para protegerse del consumo de drogas. Se ha estudiado que el afecto familiar percibido por el adolescente puede ser decisivo en el consumo de bebidas alcohólicas, a mayor afecto percibido menor consumo de bebidas alcohólicas (Uroz et al., 2018).

También se ha encontrado una relación significativa entre la funcionalidad familiar y el consumo de SPA, aquellos adolescentes que perciben a sus familias como funcio- nal tiene menor probabilidad de iniciar el consumo de drogas (Alonso-Castillo et al., 2017), e incluso se ha relacionado con otras adicciones comportamentales como el uso excesivo de internet (Cortaza-Ramírez et al., 2019).

En The Child and Family Center de la Universidad de Oregon trabajan con padres de adolescentes en el desarrollo de habilidades parentales que son importantes en la prevención y tratamiento del uso de drogas de sus hijos y en su investigación han destacado la importancia de desarrollar las siguientes aptitudes (National Institute on Drug Abuse [NIDA], 2015): 1) Comunicación, las técnicas de comunicación ayudan a los padres a detectar problemas desde muy temprano y generan un comportamiento positivo; 2) Aliento, el alentar de manera constante y sostenida a los adolescentes, les ayuda a sentirse bien con ellos mismos y les da confianza para realizar distintas actividades, mejorando su percepción de autoeficacia; 3) Negociación, negociar las soluciones ofrece un espacio para trabajar de forma conjunta padres e hijos en la resolución de problemas e incrementar la cooperación; 4) Límites, el establecimiento de límites ayuda a los padres a enseñar autocontrol y responsabilidad; 5) Supervisión, la supervisión ayuda a los padres a reconocer cuándo está surgiendo algún problema y a promover la seguridad. Las primeras cuatro aptitudes favorecen el mejoramiento de la relación padres e hijos, y la última habilidad fortalece el monitoreo paterno.

El monitoreo paterno se puede entender como los límites, reglas, restricciones y regulaciones que los padres operan para sus hijos, así como el conocimiento de las actividades que estos realizan (Betancourt y Andrade, 2011). Las investigaciones sobre el monitoreo paterno han mostrado resultados inconsistentes, por un lado, hay datos que apoyan la afirmación de que el monitoreo afecta de manera positiva la conducta de los hijos en tanto que otros estudios 
no han encontrado que le monitoreo sea un predictor para prevenir conductas de riesgo (Rodríguez et al., 2019; Voisine et al., 2008). Por su lado, se conoce que la impulsividad es un de los principales factores de riesgo que pueden predecir el inicio del consumo de drogas en adolescentes. La impulsividad es un rasgo de personalidad relevante en el estudio de las adicciones y se entiende como la tendencia a la acción sin realizar una previa valoración consciente de las consecuencias (De Sola et al., 2013; Limonero et al., 2013), frecuentemente relacionada con la búsqueda de sensaciones y la necesidad de experimentar variadas conductas de riesgo (Arunogiri et al., 2019; Gámez y Villa, 2015; Gutiérrez et al., 2013). En diferentes estudios la impulsividad es considerada un indicador para predecir el inicio y la gravedad del consumo de drogas (Gámez y Villa, 2015; Pérez-Fuentes et al., 2015), donde a mayor impulsividad mayores consecuencias negativas relacionadas a la conducta adictiva (Navas et al., 2014).

El consumo de drogas es una problemática compleja y como ya se mencionó los adolescentes son los más vulnerables, por esta razón es necesario generar conocimiento en torno a las variables que intervienen en el desarrollo de una adicción, para así diseñar intervenciones estratégicas tanto para la prevención como para el tratamiento de las adicciones en los adolescentes. En esta investigación las variables en estudio fueron la relación entre los padres y los hijos, específicamente la percepción que tiene el adolescente sobre el afecto, la comunicación, el control y la capacidad de monitoreo que el padre y la madre ejercen sobre ellos; también se midieron los niveles de impulsividad de los adolescentes.

El objetivo de esta investigación fue comparar la relación entre padres e hijos (desde las dimensiones de afecto, comunicación y control), el monitoreo paterno y materno y la impulsividad en un grupo de adolescentes estudiantes que no han consumido drogas y un grupo de adolescentes usuarios de drogas. Estos resultados podrán ayudar a establecer diferencias que puedan aportar en la comprensión de algunas variables que influyen en el desarrollo de una adicción en adolescentes.

\section{Método}

\section{Diseño}

Estudio comparativo, no experimental y de corte transversal.

\section{Participantes}

La población en estudio incluyó 90 adolescentes. 45 usuarios de drogas en tratamiento residencial y 45 estudiantes de preparatoria sin consumo de drogas. En el grupo de usuarios de drogas participaron 35 hombres y 10 mujeres con un promedio de edad de 15.40 años (DE = .539) y en el grupo de estudiantes de preparatoria participaron 19 hombres y 26 mujeres con un promedio de edad de 15.26 años (DE = 1.07).

Se seleccionó de manera intencional una comunidad terapéutica y una preparatoria pública en las cuales se tenía la posibilidad de acceder a la población en estudio. Ambo lugares están localizados en el estado de Jalisco, México. El muestreo fue no probabilístico por conveniencia.

Para el grupo de adolescentes usuarios se drogas en tratamiento residencial, los casos se seleccionaron de acuerdo a los siguientes criterios de inclusión:

- Que el adolecente aceptara de manera voluntaria participar en la investigación.

- Contar con el consentimiento informado firmado por el padre y el participante.

- Que el participante tuviera máximo cuatro semanas en tratamiento.

Para el grupo de adolescentes estudiantes de preparatoria sin consumo de drogas, los casos se seleccionaron de acuerdo a los siguientes criterios de inclusión: 
COLUNGA-RODRÍGUEZ, C., VALADEZ-GARCÍA, J., OROPEZA-TENA, R., ÁNGEL-GONZÁLEZ, M., VÁZQUEZ-COLUNGA, J., VÁZQUEZ-JUÁREZ, C., \& COLUNGA-RODRÍGUEZ, B.

- Que el adolecente aceptara de manera voluntaria participar en la investigación.

- Contar con el consentimiento informado firmado por el padre y el participante.

- Obtener solamente respuestas negativas en la subescala del POSIT que evalúa el uso de sustancias psicoactivas.

\section{Instrumentos}

Relación con Papá y Relación con Mamá (González-Forteza et al., 1999). Son dos escalas que evalúan la percepción actual que el hijo tiene de la relación que su padre y su madre establecen con él. Cada escala se compone de 15 ítems que se agrupan en las siguientes tres dimensiones: 1) el afecto que le demuestran, 2) la comunicación que establecen con él, y 3) el control que tienen de las relaciones con sus amigos. En población mexicana estas dos escalas han obtenido alfa de Cronbach de 0.93 y 0.96 , respectivamente.

Escala de Monitoreo Paterno en Adolescentes Mexicanos (Voisine et al., 2008). Este instrumento evalúa la percepción que tiene los adolescentes sobre qué tan frecuentemente los padres monitorean sus conductas. La escala consta de seis reactivos. La consistencia interna obtenida en adolescentes mexicanos fue de un alfa de Cronbach de .96 .

Escala de impulsividad (González-Forteza et al., 1997). El instrumento mide niveles de impulsividad. La escala incluye cinco reactivos y en la investigación de validación para estudiantes mexicanos la consistencia interna obtuvo un alfa de Cronbach de .70. Cuestionario de Tamizaje de los Problemas en Adolescentes (POSIT) (Mariño et al., 1998). Es un instrumento de tamizaje que contempla siete áreas de funcionamiento: uso/abuso de sustancias, salud mental, relaciones familiares, relaciones con amigos, nivel educativo, interés laboral y conducta agresiva/delincuencia. Para este estudio se utilizó la subescala de uso/abuso de sustancias la cual se compone de 17 reactivos y la adaptación en población mexicana determinó un alfa de Cronbach de .90.

\section{Variables}

Variable independiente: adolescentes con consumo de drogas y sin consumo de drogas.

Variables dependientes: la percepción que tienen los adolescentes sobre el afecto, la comunicación y el control que ejercen sus padres con ellos, el monitoreo parental y niveles de impulsividad.

\section{Procedimiento}

Primero se concertó una cita con el director de la clínica de tratamiento residencial a quien se le presentó el proyecto de investigación y aprobó su participación; después se acudió a una reunión con los adolescentes y con sus padres para explicar el proyecto y solicitar su consentimiento informado. Finalmente se programó una sesión para la aplicación de los instrumentos. Lo anterior se hizo una vez por mes durante seis meses hasta lograr la cuota de 45 participantes, ya que en esta clínica de tratamiento residencial atiende a poca población de adolescentes.

Por otro lado, se programó una cita con el subdirector de una preparatoria de la misma ciudad a quien se le presentó el proyecto de investigación y aceptó que se realizara el estudio en estudiantes de su plantel escolar. La orientadora vocacional se encargó de seleccionar un grupo de cada grado escolar para aplicar la subescala del POSIT y una vez que se obtuvieron los resultados, se le eligieron por conveniencia a 45 estudiantes que hayan obtenido respuestas negativas en todos los ítems de la subescala del POSIT. La orientadora vocacional envío a los padres de los estudiantes seleccionados un comunicado con la información de la investigación y el consentimiento informado para ser firmado por ellos y sus hijos en caso de aceptar participar en el estudio. Una vez que se recabaron todos los con- 
sentimientos informados se programó una fecha para aplicar los instrumentos de evaluación de manera grupal en una sola jornada.

Después de haber recolectado toda la información de las poblaciones en estudio se procedió a la captura de los datos para realizar el procesamiento, análisis y conclusiones pertinentes.

\section{Análisis de datos}

El análisis estadístico de los datos se llevó a cabo mediante el programa IBM SPSS $v$. 21 para Windows. Se determinó el nivel alfa de significancia estadística $(\alpha)$ en 0.05 y se realizó un análisis de diferencia de las variables relación padre-hijo, relación madre-hijo, monitoreo paterno, monitoreo materno e impulsividad entre dos grupos por medio de la t de Student para grupos independientes.

\section{Consideraciones éticas}

De acuerdo con la American Psychological Association (2017) y el Código Ético del Psicólogo (Sociedad Mexicana de Psicología, 2009) se identificó que esta investigación no genera ningún riesgo para los participantes. Todos los participantes aceptaron contestar los instrumentos voluntariamente y sus padres otorgaron el consentimiento informado. Se enfatizó en la confidencialidad de la información.

\section{Resultados y Discusión}

En la Tabla 1 se presentan los resultados de la percepción que los adolescentes tienen en torno a la relación padre-hijo y sus dimensiones afecto, comunicación y control, todas indican una diferencia estadísticamente significativa entre los grupos en estudio, con excepción de la dimensión afecto paterno. Lo mismo sucede con la variable relación madre-hijo y sus dimensiones afecto, comunicación y control, todas muestran una diferencia estadísticamente significativa entre los grupos en estudio, con excepción de la dimensión afecto materno.

Para todas las variables el grupo de adolescentes estudiantes obtuvo mayores puntajes en comparación al grupo de adolescentes usuarios de drogas.

Tabla 1. Resultado de la prueba de diferencia de las variables relación padre-hijo y relación madre-hijo

\begin{tabular}{|c|c|c|c|c|c|}
\hline \multirow[t]{2}{*}{ Variables } & $\begin{array}{c}\text { Adolescentes } \\
\text { estudiantes }\end{array}$ & $\begin{array}{l}\text { Adolescentes } \\
\text { usuarios de drogas }\end{array}$ & \multirow[t]{2}{*}{$t$} & \multirow[t]{2}{*}{$g l$} & \multirow{2}{*}{$p$} \\
\hline & $\overline{\bar{X}}(D E)$ & $\overline{\bar{X}}(D E)$ & & & \\
\hline Relación padre-hijo & $44.60(11.48)$ & $34.13(8.10)$ & 4.99 & 88 & .000 \\
\hline Dimensión afecto paterno & $16.62(4.35)$ & $15.09(3.73)$ & 1.79 & 88 & .076 \\
\hline Dimensión comunicación paterna & $12.60(4.41)$ & $8.47(2.95)$ & 5.21 & 88 & .000 \\
\hline Dimensión control paterno & $15.38(3.63)$ & $10.58(2.83)$ & 6.98 & 88 & .000 \\
\hline Relación madre-hijo & $49.24(10.39)$ & $39.04(9.53)$ & 4.85 & 88 & .000 \\
\hline Dimensión afecto materno & $17.71(3.94)$ & $16.60(3.95)$ & 1.33 & 88 & .185 \\
\hline Dimensión comunicación materna & $14.98(4.25)$ & $11.07(3.67)$ & 4.66 & 88 & .000 \\
\hline Dimensión control materno & $16.56(3.19)$ & $11.38(3.11)$ & 7.78 & 88 & .000 \\
\hline
\end{tabular}


COLUNGA-RODRÍGUEZ, C., VALADEZ-GARCÍA, J., OROPEZA-TENA, R., ÁNGEL-GONZÁLEZ, M., VÁZQUEZ-COLUNGA, J., VÁZQUEZ-JUÁREZ, C., \& COLUNGA-RODRÍGUEZ, B.

Por otro lado, en la Tabla 2 se presentan los resultados de la prueba de diferencias para las variables monitoreo paterno, monitoreo materno e impulsividad. En todas las variables se encontró una diferencia estadísticamente significativa entre los grupos en estudio.
Para las variables monitoreo paterno y monitoreo materno, el grupo de adolescentes estudiantes obtuvo mayores puntajes en comparación al grupo de adolescentes usuarios de drogas y en el caso de la variable impulsividad los adolescentes usuarios de drogas obtuvieron puntajes mayores en comparación al grupo de adolescentes estudiantes.

Tabla 2. Resultado de la prueba de diferencia de las variables monitoreo paterno y materno e impulsividad

\begin{tabular}{lcccccc}
\hline \multicolumn{1}{c}{ Variable } & $\begin{array}{c}\text { Adolescentes } \\
\text { estudiantes }\end{array}$ & $\begin{array}{c}\text { Adolescentes } \\
\text { usuarios de drogas }\end{array}$ & $t$ & $g l$ & $p$ \\
\cline { 2 - 4 } & $\overline{\bar{X}}(\mathrm{DE})$ & $\overline{\bar{X}}(\mathrm{DE})$ & & & \\
\hline Monitoreo paterno & $18.33(4.04)$ & $13.71(2.94)$ & & 6.20 & 88 & .000 \\
Monitoreo materno & $19.91(2.87)$ & $14.69(3.64)$ & & 7.54 & 88 & .000 \\
Impulsividad & $10.24(3.65)$ & $14.09(4.00)$ & -4.75 & 88 & .000 \\
\hline
\end{tabular}

En lo referente a las variables relación padre-hijo y madre-hijo, en el grupo de adolescentes estudiantes se encontró un mayor puntaje en las dimensiones comunicación y control en comparación con el grupo de adolescentes usuarios de drogas. Por lo anterior podemos deducir que la comunicación y el control paterno y materno, probablemente son factores de protección en tanto que se presentan mayores niveles en el grupo de estudiantes, así mismo podemos inferir que la falta de comunicación y de control paterno y materno pueden ser factores de riesgo, en tanto que se presentan menores niveles en el grupo de usuarios de drogas. Resultados similares se encontraron en un estudio que refiere que una deficiente relación de los jóvenes con sus padres aumenta la probabilidad de iniciar el consumo de drogas (Martínez et al., 2013; Pérez-Hernández et al., 2016).

La falta de comunicación familiar se ha estudiado como una de las principales posibles causas para desarrollar una adicción (Rivadeneira et al., 2020; Ruiz et al., 2017)), así, la falta de comunicación dificulta que los padres se enteren de los eventos ad- versos por los que sus hijos transitan y esto incrementa la probabilidad de desarrollar conductas de riesgo.

Una de las características de la etapa de la adolescencia es la tendencia a mostrar poco interés por el cumplimiento de normas, en ese sentido los padres necesitan desarrollar un adecuado control parental partiendo de las necesidades de sus hijos y centrándose en el cumplimiento de normas y responsabilidades (Challco et al., 2016; Moreno y Palomar, 2017). El bajo control parental se ha relacionado con una alta permisividad familiar y como posible consecuencia el consumo de drogas en los adolescentes (Moreno y Palomar, 2017; Pérez-Hernández et al., 2016; Ruiz et al., 2017), por esta razón es importante que las intervenciones preventivas familiares incluyan tópicos encaminados a mejorar la comunicación familiar y el control parental (NIDA, 2015; Orte y Ballester, 2018).

Para la dimensión de afecto paterno y afecto materno, no se encontraron diferencias significativas entre los grupos en estudio, estos resultados son contrarios a varias in- 
vestigaciones que refieren que la carencia afectiva por parte de los padres se asocia a un mayor riesgo de desarrollar una adicción en los adolescentes (Alonso-Castillo et al., 2017; Cortaza-Ramírez et al., 2019; Uroz et al., 2018), sin embargo en otro estudio se encontró que ni la estructura familiar ni el tiempo que los padres le dedican a sus hijos está relacionado con el consumo de drogas (Moreno y Palomar, 2017). Es importante destacar que la investigación que apoya la hipótesis de que a menor afecto de los padres mayor consumo de drogas en los hijos, se realizaron en una sola población de estudiantes; por otro lado, esta investigación se llevó a cabo en dos poblaciones independientes, adolecentes estudiantes que no han usado drogas y adolescentes usuarios de drogas en tratamiento residencial.

En una lectura detallada de las medias de las tres dimensiones de las variables de relación padre-hijo y madre-hijo, se encontró los siguiente: 1) las dimensiones de menor puntaje en el grupo de usuarios de drogas fueron la comunicación y el control, por lo que es fundamental considerar estas variables en el momento de diseñar intervenciones preventivas; 2) en ambos grupos en estudio las madres obtuvieron mayor puntaje que los padres en todas las dimensiones (afecto, comunicación y control). Otras investigaciones refieren que las madres suelen tener vínculos relacionales más cercanos y que los padres generalmente se mantienen periféricos, así, a la mujer se le ha asignado culturalmente el cuidado de los hijos (Szapocznik et al., 2003; Zapata, 2015).

El monitoreo parental se puede entender como los límites y regulaciones que los padres operan para sus hijos, así como el conocimiento de las actividades que estos realizan (Betancourt y Andrade, 2011). En este estudio, se encontró una diferencia estadísticamente significativa en la variable monitoreo paterno y el monitoreo materno, donde el grupo de adolescentes estudian- tes obtuvo mejore puntaje. Estos resultados coincidieron con otras investigaciones que señalan que un buen monitoreo o supervisión de los hijos es un factor de protección (De Avila-Arroyo et al., 2019; Schinke et al., 2016). Los padres que tienen mayor capacidad de monitoreo pueden proteger a los jóvenes del consumo de drogas y por lo tanto el no contar con un monitoreo adecuado y no establecer límites claros a los jóvenes puede resultar en un riesgo para iniciar el consumo de drogas (NIDA, 2015).

En cuanto a los niveles de impulsividad se encontraron diferencias estadísticamente significativas entre los dos grupos en estudio. En diversas investigaciones se ha encontrado que la impulsividad está presente en los jóvenes consumidores de drogas, donde su capacidad de autocontrol es limitada, aumentando la probabilidad de que los jóvenes desarrollen conductas de riesgo e inicien el consumo de drogas (Arunogiri et al., 2019; Gámez y Villa, 2015; Gutiérrez et al., 2013; Pérez-Fuentes et al., 2015).

La etapa de la adolescencia se considera la de mayor riesgo para comenzar el consumo de SPA, ocasionando diferentes daños a la salud que impactan en su desarrollo individual, familiar y social (Jenkins, 2017; Stanis y Andersen, 2014; United Nations Office on Drugs and Crime [UNODC], 2015). El inicio temprano del consumo de SPA está relacionado con la posible futura adquisición de una dependencia, debido a la vulnerabilidad biológica, psicológica y social en que se encuentran los adolescentes, por esta razón es importante el estudio de las variables que se relacionan con el consumo de SPA en adolescentes y así contar con elementos para diseñar intervenciones preventivas eficaces dirigidas tanto para los adolescentes como para sus familias (Valadez-García et al., 2020; Valadez-García y Oropeza-Tena, 2020). 
COLUNGA-RODRÍGUEZ, C., VALADEZ-GARCÍA, J., OROPEZA-TENA, R., ÁNGEL-GONZÁLEZ, M., VÁZQUEZ-COLUNGA, J., VÁZQUEZ-JUÁREZ, C., \& COLUNGA-RODRÍGUEZ, B.

\section{Conclusiones}

A manera de conclusión se puede decir que los padres de los adolescentes usuarios de drogas cuentan con pocas aptitudes para lograr afrontar la etapa de la adolescencia y los riesgos que esto conlleva. Es así, que los padres de jóvenes con altos niveles de impulsividad necesitan realizar un esfuerzo extraordinario para mejorar su capacidad de control parental y las estrategias de comunicación, sin descuidar los aspectos afectivos y poder prevenir el uso de drogas. Por otro lado, en el caso de jóvenes con bajos niveles de impulsividad tanto el control parental, la comunicación y el afecto deben de implementarse de forma equilibrada. Estas importante comprender que estas tres dimensiones se influyen mutuamente.

Finalmente, es importante que los padres estén atentos al tipo de amistades con quien se relacionan los hijos, monitoreando con eficacia, pero con la suficiente distancia para fomentar la autonomía y desarrollo de identidad (NIDA, 2015). Los resultados de esta investigación pueden ser de utilidad para el diseño de intervenciones preventivas, las cuales deben estar fundamentadas con base en la literatura científica, así como en los estándares internacionales (UNODC, 2015).

\section{Limitaciones}

Los resultados de la presente investigación deben tomarse con cierta cautela, puesto que es un estudio que se realizó con una población finita y se debe continuar explorando en otros contextos y determinar la direccionalidad de las variables. Otra de las limitaciones es la correspondiente al tamaño de la muestra y al tipo de muestreo. Además, se tuvo un sesgo muestral, debido a que el estudio se realizó solamente en una escuela preparatoria y en una sola clínica de tratamiento residencial, sin embargo, existen muy pocas clínicas que brinden tratamiento a adolescentes consumidores de drogas.

\section{Financiamiento}

Para la elaboración del presente artículo se contó con el apoyo económico del Consejo Nacional de Ciencia y Tecnología (CONACYT).

\section{Conflicto de interés}

Los autores declaran no tener ningún conflicto de interés.

\section{Bibliografía}

Alonso-Castillo, M.M., Yañez-Lozano, A., \& Armendáriz-García, N.A. (2017). Funcionalidad familiar y consumo de alcohol en adolescentes de secundaria. Health and Addictions, 17(1), 87-96. http://doi. org/10.21134/haaj.v17i1

American Psychological Association (2017). Ethical principles of psychologists and code of conduct. APA 125. https://www.apa.org/ethics/code/ ethics-code-2017.pdf

Arunogiri, S., Verdejo-Garcia, A., McKetin, R., Rubenis, A.J., Fitzpatrick, R., \& Lubman, D.I. (2019). Emotion recognition and impulsive choice in relation to methamphetamine use and psychosis symptoms. Frontiers in Psychiatry, 10, 889. http:// doi.org/10.3389/fpsyt.2019.00889

Betancourt, D., \& Andrade, P.P. (2011). Control parental y problemas emocionales y de conducta en adolescentes. Revista Colombiana de Psicología, 20(1), 27-41.

Challco, K., Rodríguez, S., \& Jaimes, J. (2016). Riesgo de adicción a redes sociales, autoestima y autocontrol en estudiantes de secundaria. Revista Científica de Ciencias de la Salud, 9(1), 9-15. https://doi.org/10.17162/rccs.v9i1.542

Cortaza-Ramírez, L., Blanco-Enríquez, F., Hernández-Cortaza, B.A., Lugo-Ramírez, L.A., Beverido, P., Salas, B., \& Jorge-Cárdenas, X. (2019). Uso de internet, consumo de alcohol y funcionalidad familiar en adolescentes mexicanos. Health and Addictions, 19(2), 59-69. http://doi.org/10.21134/ haaj.v19i2

De Avila-Arroyo, M.L., Rodríguez-Aguilar, L., Armendáriz-García, N.A., Pérez-Carrillo, V.E., Tenahua-Quitl, I., \& Guzmán-Facundo, F.R. (2019). Factores de riesgo y etapas de adquisición del consumo de drogas lícitas en estudiantes mexicanos. Journal Health NPEPS, 4(2), 280-296. http:// doi.org/10.30681/252610103761 
De Sola, J., Rubio, G., \& Rodríguez, F. (2013). La impulsividad: ¿antesala de las adicciones comportamentales? Health and Addictions, 13 (2), 145-155.

Figueroa-Varela, M.R., Lira-Renteria, S.G., \& González-Betanzos, F. (2019). Factores de riesgo para el consumo de alcohol y drogas en estudiantes de bachillerato en Nayarit, México. Health and Addictions, 19(2), 130-138. http://doi.org/10.21134/haaj. v19i2

Gámez, M., \& Villa, F.I. (2015). El modelo cognitivo-conductual de la adicción a internet: el papel de la depresión y la impulsividad en adolescentes mexicanos. Psicología y Salud, 25(1), 111-122.

González-Forteza, C., Andrade, P., \& Jiménez, A. (1997). Recursos psicológicos relacionados con el estrés cotidiano en una muestra de adolescentes mexicanos. Salud Mental, 20 (1), 27-35.

González-Forteza, C., Jiménez, A., Pérez, E., Ramos, L., Caballero, M.A., \& Saltijeral, M.T. (1999). Padres afectivos: apoyo para la autoestima de sus hijos adolescentes. Psicología Conductual, 7(3), 501-507.

Gutiérrez, J., Rubio, G., \& Rodríguez, F. (2013). Impulsivity: the prelude to behavioral addictions? Health and Addictions, 13 (2), 145-155.

Jenkins, E.K., Slemon, A., \& Haines-Saah, R.J. (2017). Developing harm reduction in the context of youth substance use: insights from a multi-site qualitative analysis of young people's harm minimization strategies. Harm Reduction Journal 14(53), 1-11. http://doi.org/10.1186/s12954-017-0180-z

Limonero, J., Gómez-Romero, M.J., Fernández-Castro, J., \& Tomás-Sábado, J. (2013). Influencia de la inteligencia emocional percibida y la impulsividad en el abuso de cánnabis en jóvenes. Ansiedad y Estrés, 19(3), 223-234.

Mariño, M.C., Gonzáles-Forteza, C., Andrade, P., \& Medina-Mora, M.L. (1998). Validación de un cuestionario para detectar adolescentes con problemas por el uso de drogas. Salud Mental, 21(1), 27-36.

Martínez, I., Fuentes, M.C., García, F., \& Madrid, I. (2013). El estilo de socialización familiar como factor de prevención o riesgo para el consumo de sustancias y otros problemas de conducta en los adolescentes españoles. Adicciones, 25(3), 235242. http://doi.org/10.20882/adicciones.51

Moreno, N.D., \& Palomar, J. (2017). Factores familiares y psicosociales asociados al consumo de drogas en adolescentes. Interamerican Journal of Psychology, 51(2), 141-151.
National Institute on Drug Abuse (2015). Family checkup: positive parenting prevents drug abuse. National Institute of Health.

Navas, J.F., Torres, A., Cándido, A., \& Perales, J.C. (2014). ¿Nada o un poco? ¿Mucho o demasiado? La impulsividad como marcador de gravedad en niveles problemático y no problemático de uso de alcohol e Internet. Adicciones, 26 (2), 159-167.

Orte, C., \& Ballester, L. (2018). Intervenciones efectivas en prevención familiar de drogas. Octaedro.

Pérez-Fuentes, M.C., Gázquez, J.J., Molero, M., Cardila, F., Martos, A., Barragán, A.B., Garzón, A., Carrión, J.J., \& Mercader, I. (2015). Impulsividad y consumo de alcohol y tabaco en adolescentes. European Journal of Investigation in Health, Psychology and Education, 5(3), 371-382. http://doi. org/10.1989/ejihpe.v5i3.139

Pérez-Hernández, E.A., Mendieta, G., \& Nuño, B.L. (2016). Dimensión estructural: configuración familiar de adolescentes fumadores en una preparatoria pública de Guadalajara, México. Investigaciones Andina, 32(18), 1480-1490.

Rivadeneira, Y.M., Cajas, T.A., Viejó, I., \& Quinto, E. (2020). Adicciones a sustancias y comportamentales en la ciudad de Loja. Revista Psicología UNEMI, 4(6), 20-29.

Rodríguez, A., Rico, D., Montero, O.L., \& Rubio, A.M. (2019). Relación del consumo de alcohol y el monitoreo parental con el inicio de las relaciones sexuales de los adolescentes escolarizados de Colombia. Revista Academia y Virtualidad, 12(2), 95-106. https://doi.org/10.18359/ravi.4338

Ruiz, L.D., Gayoso, M., \& Prada, R.E. (2017). Funcionamiento familiar y factores de riesgo de consumo de drogas en adolescentes de un Centro Juvenil Penitenciario. Revista Paian, 8(2), 13-28.

Schinke, S., Schwinn, T., Hopkins, J., \& Wahlstrom, L. (2016). Drug abuse risk and protective factors among Hispanic adolescents. Preventive Medicine Reports, 3, 185-188. http://doi.org/10.1016/j. pmedr.2016.01.012

Sociedad Mexicana de Psicología (2009). Código Ético del Psicólogo. Trillas.

Stanis, J., \& Andersen, S. (2014). Reducing substance use during adolescence: a translational framework for prevention. Psychopharmacology, 231(8), 1437-1453.

Szapocznik, J., Hervis, O., \& Scwartz, S. (2003). Brief strategic family therapy for adolescent drug abuse. National Institute of Health. 
United Nations Office on Drugs and Crime (UNODC) (2015). International standards on drug use prevention. United Nations Publication.

Uroz, J., Charro, B., Prieto, M., \& Meneses, C. (2018). Estructura familiar y consumo de alcohol en adolescentes. Health and Addictions, 18(1), 107-118. http://doi.org/10.21134/haaj.v18i1

Valadez, J.A., Oropeza, R., Salazar, M.L., \& Martínez, K. (2018). La voz de los profesionales: componentes y sugerencias para los programas de prevención en adicciones. Revista Electrónica de Psicología Iztacala, 21(3), 796-816.

Valadez-García, J.A., \& Oropeza-Tena, R. (2020). Evaluación de la Intervención Preventiva para Estudiantes Adolescentes en Riesgo (IPEA-R) en estudiantes de secundaria. Health and Addictions, 20(2), 157-169. https://doi.org/10.21134/haaj. v20i2

Valadez-García, J.A., Oropeza-Tena, R., Salazar-Garza, M.L., \& Fulgencio-Juárez, M. (2020). Desarrollo de intervenciones para prevenir el consumo de drogas en estudiantes adolescentes. En M.G., Luna; \& R., Montes (comp.). Investigación Interinstitucional en Psicología. Nuevos desafíos para el siglo XXI (91-110). Grañén Porrúa.

Villatoro-Velázquez J.A., Bustos, M., Oliva, N., Fregoso, D., Mujica, A., Martín del Campo, R., Nanni, R. \& Medina-Mora M.E. (2015a). Encuesta Nacional de Consumo de Drogas en Estudiantes 2014: Reporte de Tabaco. Instituto Nacional de Psiquiatría Ramón de la Fuente Muñiz; Comisión Nacional Contra las Adicciones, Secretaría de Salud. https:// www.gob.mx/salud\%7Cconadic/documentos/encuesta-nacional-de-consumo-de-drogas-en-estudiantes-2014-encode
Villatoro-Velázquez J.A., Fregoso, D., Bustos, Oliva, N., Mujica, A., Martín del Campo, R., Nanni, R. \& Medina-Mora M.E. (2015b). Encuesta Nacional de Consumo de Drogas en Estudiantes 2014: Reporte de Alcohol. Instituto Nacional de Psiquiatría Ramón de la Fuente Muñiz; Comisión Nacional Contra las Adicciones, Secretaría de Salud. https:// www.gob.mx/salud\%7Cconadic/documentos/encuesta-nacional-de-consumo-de-drogas-en-estudiantes-2014-encode

Villatoro-Velázquez J.A., Oliva, N., Fregoso, D., Bustos, M., Mujica, A., Martín del Campo, R., Nanni, R. \& Medina-Mora M.E. (2015c). Encuesta Nacional de Consumo de Drogas en Estudiantes 2014: Reporte de Drogas. Instituto Nacional de Psiquiatría Ramón de la Fuente Muñiz; Comisión Nacional Contra las Adicciones, Secretaría de Salud. https:// www.gob.mx/salud\%7Cconadic/documentos/encuesta-nacional-de-consumo-de-drogas-en-estudiantes-2014-encode

Voisine, S., Parsai, M., Marsiglia, F., Kulis, S., \& Nieri, T. (2008). Effects of parental monitoring, permissiveness, and injunctive norms on substance use among Mexican and Mexican American adolescents. Families in Society, 89(2), 264-273. http:// doi.org/10.1606/1044-3894.3742

Zapata, A. (2015). Madres y padres en contextos transnacionales: el cuidado desde el género y la familia. Desacatos, (52), 14-31.

\section{CitAR ESTE ARTICULO:}

Colunga-Rodríguez, C., Valadez-García, J., Oropeza-Tena, R., Ángel-González, M., Vázquez-Colunga, J., Vázquez-Juárez, C., \& Colunga-Rodríguez, B. (2021). Impulsividad, monitoreo y relación parental entre adolescentes estudiantes y adolescentes usuarios de drogas. RECIMUNDO, 5(4), 284-294. ht-

tps://doi.org/10.26820/recimundo/5.(4).oct.2021.284-294 (c) (1) (\$) (2) BY NC SA

CREATIVE COMMONS RECONOCIMIENTO-NOCOMERCIAL-COMPARTIRIGUAL 4.0 . 